ficacy, usually involving destructive or excisional procedures. Significant research activity is currently focused on developing both prophylactic and therapeutic vaccines against HPV.

Therapeutic vaccine efforts are mostly based on the HPV oncoproteins E6 and E7, which are selectively retained and expressed in cervical tumors associated with HPV 16. Recently the first phase I/II clincal trial of a live recombinant vaccinia virus expressing the E6 and E7 proteins of HPV 16 and HPV 18 tested safety and immunogenicity in eight patients with late-stage cervical cancer ${ }^{7}$. No side effects were noted and three of eight patients mounted an HPV-specific antibody response. HPV-specific cytotoxic T lymphocytes, thought to be the most therapeutically beneficial effector mechanism, were detected in one patient. Although the study was too small to draw firm conclusions, the encouraging results warrant further investigation in a larger clinical trial.

Prophylactic vaccine development has focused on recombinant subunit preparation consisting of the L1 and L2 virion structural proteins, but recently it has been shown that the HPV L1 and L2 capsid proteins coassemble into papillomavirus-like particles when present in high levels in eukaryotic cells. A recent report by Suzich et $a .^{8}$ has described a successful canine model in which injection of the L1 capsid protein completely protected beagles from experimental challenge with canine oral papillomavirus. The future development of both prophylactic and therapeutic vaccines for HPV is expected to offer an effective alternative to expensive screening programs for the prevention of cervical cancer.

1. National Institutes of Health, Consensus Development Conference Statement. Cervical Cancer. April 1-3, 1996.
2. Parker, S.L., Tong, T., Bolden, S. \& Wingo, P.A. Cancer statistics, 1996. CA $-A$ Cancer I. Clinicians 46, 5-27 (1996)

3. Hayward, R.A. et al. Who gets screened for cervical and breast cancer? Results from a new national survey. Arch. Intern. Med. 148, 1177-1181 (1988).

4. Mandelblatt, J. et al. Gynecological care of elderly women: Another look at Papanicolaou smear testing. JAMA 256, 367-371 (1986).

5. Lehtinen, M. et al. Serologically diagnosed infection with human papillomavirus type 16 and risk for subsequent development of cervical carcinoma: Nested case-control study. Br. Med. J. 2 , 537-539 (1996).

6. Prokopczyk, B. et al. Identification of tobaccospecific carcinogens in the cervical mucus of smokers and nonsmokers. Proc. Am. Assoc. Cancer Res. 37, Abstr. 100 (1996).

7. Borysiewicz, L.K. et al. A recombinant vaccinia virus encoding human papillomavirus types 16 and 18, E6 and E7 proteins as immunotherapy for cervical cancer. Lancet 347, 1523-1527 (1996).

8. Suzich, J.A. et al. Systemic immunization with papillomavirus LI protein completely prevents the development of viral mucosal papillomas. Proc. Natl. Acad. Sci. USA 92, 11553-11557 (1995).

Department of Obstetrics and Gynecology Louisiana State University Medical Center New Orleans, Lousiana, 70112, USA

\title{
Progress towards a vaccine to prevent sexual transmission of HIV
}

\author{
Immunization with subunit antigens prevents rectal transmission of SIV in monkeys (pages 767-775).
}

Sexual transmission is the most common route for dissemination of human immunodeficiency virus (HIV) worldwide, and a vaccine capable of preventing sexual transmission might prove an effective way of halting the spread of AIDS. To be effective such a vaccine must prevent HIV transmission by three distinct mucosal routes: oral, rectal and vaginal. Each of these mucosal surfaces has a distinct mucosa-associated lymphoid tissue, and it is possible that the induction of immunity and the nature of the cellular and molecular immune responses may differ at each of these sites. Interest in developing HIV vaccines that elicit immune responses at mucosal surfaces has been spurred by studies showing that the same immunization protocols that protect monkeys from intravenous challenge with simian immunodeficiency virus (SIV) do not provide protection from vaginal SIV challenge ${ }^{1}$. Likewise, the SIV-vaginal infection model suggests that the route of dissemination after mucosal exposure to SIV involves infection of antigen-presenting cells (APCs, both Langerhans cells and macrophages) in the genital mucosa followed by a stepwise progression of the infection from regional lymph nodes to distant lym-

\section{CHRISTOPHER J. MILLER ${ }^{1} \&$ JERRY R. MCGHEE ${ }^{2}$}

phoid tissues, presumably by migration of infected APCs (ref. 2, 3; see figure). These observations have led to the hypothesis that systemic immunity alone cannot prevent the genital transmission of SIV in monkeys or HIV in humans.

Two studies have shown protection of monkeys from rectal transmission after intravenous inoculation with a "subinfectious dose" of SIV (ref, 4) or intramuscular immunization with human $\mathrm{T}$ cell-derived whole-killed SIV (ref. 5) (although in the latter study protection may have been due to xenogeneic immune responses). In this issue of Nature Medicine Lehner et al. ${ }^{6}$ significantly extend these earlier studies. They report prevention of rectal transmission of SIV in macaques using an SIV subunit vaccine and an immunization strategy designed to elicit mucosal immune responses. By taking advantage of the lymphatic drainage from the rectum, the authors were able to use a nonmucosal immunization route to generate strong anti-SIV mucosal immunity. A similar approach has been used in mice to elicit genital tract immunity ${ }^{7}$, but the report by Lehner et al. is the first to show that immune responses generated by this route are effective against rectal transmission of SIV.

In two series of experiments macaques received a recombinant SIV vaccine (composed of envelope gp120 and core gag p27 antigens) by injection into the tissues around the iliac lymph nodes, which drain the rectal mucosa. After rectal SIV challenge, the authors report complete protection in four out of seven monkeys (and reduced virus load in the remainder) compared with infection in 13 out of 14 control animals. This is the first demonstration that protection from rectal challenge can be achieved with a vaccine composed of subunit antigens in monkeys. Earlier monkey studies used whole-killed or live-attenuated SIV vaccines but, because of safety concerns, such an approach may be unsuitable for HIV vaccines. A recent serological analysis of HIV-1 isolates from different clades shows that serum antibodies from HIV-1-infected individuals react to envelope proteins of HIV-1 isolates, which are genetically very divergent from the original infective virus ${ }^{8}$. This encouraging report suggests that if the right 


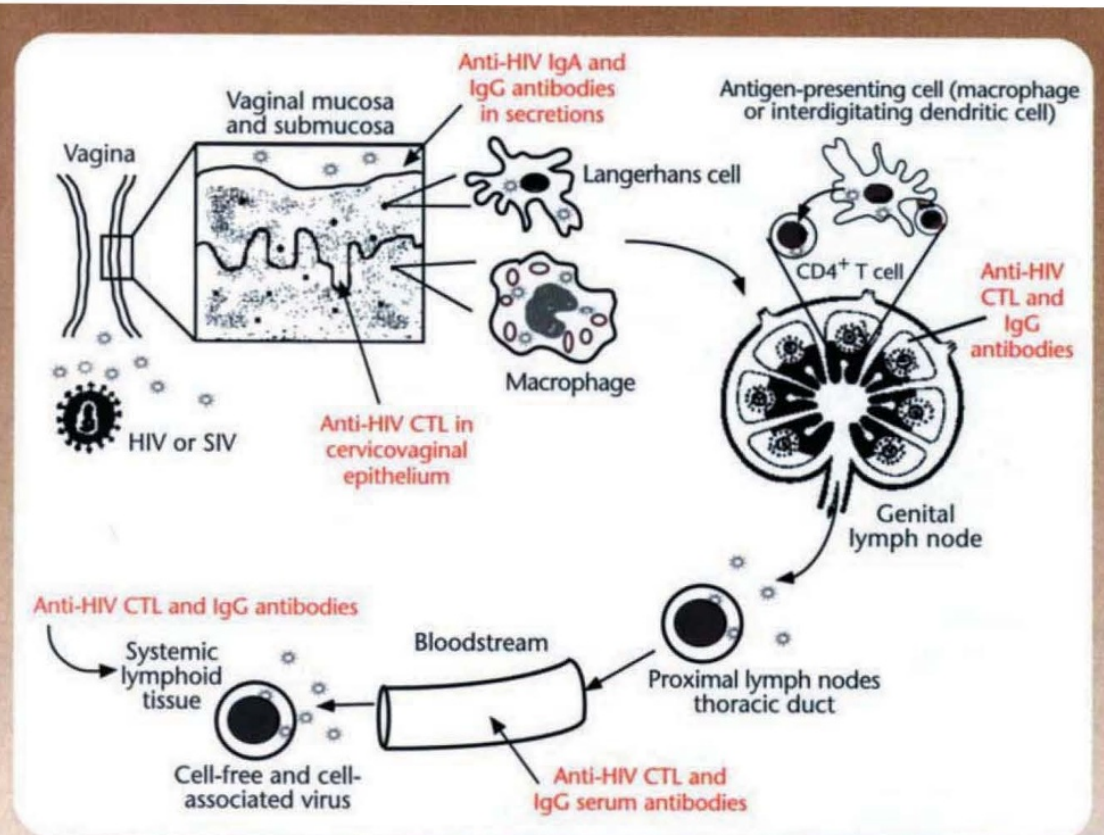

HIV dissemination following sexual transmission. Virus infects submucosal antigen-presenting cells that travel to draining lymph nodes where further viral replication occurs. The virus then enters the circulation and is disseminated to systemic lymphoid tissues. Immune effector mechanisms (in red), capable of controlling virus replication or eliminating infected cells, are present in all of these anatomic sites (adapted from ref. 3 ).

envelope antigens are used in a vaccine, it may be possible to elicit effective broad anti-HIV immunity.

Although the direct injection of connective tissue surrounding the iliac lymph nodes is unlikely to be used in a clinical setting, Lehner's group are also pursuing conventional subcutaneous and/or intramuscular routes of immunization with the same vaccine (personal communication). The authors propose that vaccineinduced $\mathrm{CD}^{+} \mathrm{T}$ cell-mediated suppression of viral replication may account for the observed protection. However, it is not clear that this suppression was mediated by the viral antigens in the vaccine, as one of the naive control animals also had high levels of activity. Other investigators have demonstrated SIV suppressor activity in SIV-naive monkeys (B.L. Lohman, personal communication) and $\mathrm{CD}^{+} \mathrm{T}$-cell HIV suppressor activity has been found in seronegative humans ${ }^{9}$. Furthermore, $\mathrm{CD} 8^{+}$ T-cell viral suppression in HIV-1-infected individuals does not appear to be lentivirus-specific ${ }^{10}$. This raises the intriguing possibility that stimulating nonHIV-specific immune responses may protect individuals from rectal challenge.

The results of Lehner et al. using the SIV/monkey model suggest that it may be possible to produce a vaccine to pre- vent rectal transmission of HIV. However, it is clear that it will be substantially more difficult to produce a vaccine to prevent HIV transmission in the female reproductive tract. The targeted lymph node immunization strategy of Lehner's group has, thus far, not provided reliable protection from vaginal challenge with highly pathogenic SIV. Several groups are developing vaccines to prevent sexual transmission of SIV by other mucosal routes. A parenterally administered live-attenuated vaccine protects against oral transmission of SIV in young adult macaques ${ }^{11}$. Although parenterally administered, attenuated and killed vaccines do not prevent vaginal SIV transmission, a vaginally administered attenuated vaccine does provide protection from vaginal challenge with SIV (C.J.M., unpublished). Perhaps the $\mathrm{SIV} /$ monkey model of AIDS is best used to accurately define the immunological mechanisms by which animals vaccinated with attenuated vaccines are protected from challenge with virulent SIV.

If the current paradigm of stepwise dissemination of HIV from mucosal surface to regional lymph nodes and then to systemic lymphoid tissue is correct, then the best vaccine strategy will be to elicit antiHIV immune responses at each site of virus entry, replication and dissemination (see figure). The fact that SIV-specific cytotoxic T lymphocytes have now been observed in the vaginal mucosa of monkeys $^{12}$ implies that a full range of antiviral immunity can be brought to bear in an attempt to blunt each stage of virus transmission and dissemination. Identifying viral antigens and immunization routes that can elicit these immune responses and determining which of these can be applied in a clinical setting remain the challenge before us.

1. Marthas, M. et al. Efficacy of live-attenuated and whole-inactivated simian immunodeficiency virus vaccines against intravenous and intravaginal challenge. in Vaccines 1992 (ed. Brown, F., Chanock, R.M., Ginsberg, H.S. \& Lerner, R.A.) 117-122 (Cold Spring Harbor Laboratory Press, Cold Spring Harbor, New York, 1992).

2. Miller, C.J., Alexander, N.J., Vogel, P., Anderson, J. \& Marx, P.A. Mechanism of genital transmission of SIV: A hypothesis based on transmission studies and the location of SIV in the genital tract of chronically infected female rhesus macaques. J. Med. Primatol. 21, 64-68 (1992).

3. Miller, C.J., McGhee, J.R. \& Gardner, M.B. Mucosal immunity, HIV transmission and AIDS. Lab. Invest. 68, 129-145 (1992).

4. Clerici, M. et al. T-cell proliferation to subinfectious SIV correlates with lack of infection after challenge of macaques. AIDS 8, 1391-1395 (1994).

S. Cranage, M.P. et al. Intrarectal challenge of macaques vaccinated with formalin-inactivated simian immunodeficiency virus. Lancet 339 , 273-274 (1992).

6. Lehner, T. et al. Protective mucosal immunity elicited by targeted lymph node immunization with a subunit SIV envelope and core vaccine in macaques. Nature Med. 2, 767-775 (1996).

7. Thapar, M.A., Parr, E.L. \& Parr, M.B. Secretory immune responses in mouse vaginal fluid after pelvic, parenteral or vaginal immunization. Immunology 70, 121-125 (1990).

8. Moore, J.P. et al. Inter- and intraclade neutralization of human immunodeficiency virus type 1: Genetic clades do not correspond to neutralization serotypes but partially correspond to gp120 antigenic serotypes. J. Virol. 70, 427-444 (1996).

9. Brinchmann, J.E., Gaudernack, G. \& Vartdal, F. $\mathrm{CD} 8+\mathrm{T}$ cells inhibit HIV replication in naturally infected CD4+ $\mathrm{T}$ cells. J. Immunol. 144, 2961-2966 (1990).

10. Copeland, K.F.T., McKay, P.J. \& Rosenthal, K.L. Suppression of activation of the human immunodeficiency virus long terminal repeat by CD8+ $\mathrm{T}$ cells is not lentivirus specific. AIISS Res. Hum. Retrovir. 11, 1321-1326 (1995).

11. Otsyula, M.G. et al. Fetal or neonatal infection with attenuated simian immunodeficiency virus results in protective immunity against oral challenge with pathogenic SIVmac251. Virology (in the press).

12. Lohman, B.L., Miller, C.J. \& McChesney, M.B. Antiviral cytotoxic $\mathrm{T}$ lymphocytes in the vaginal mucosa of simian immunodeficiency virusinfected rhesus macaques. I. Immunol. 155, 5855-5860 (1995).

${ }^{1}$ Califormia Regional Primate Research Center University of California Davis

Davis, California 95616, USA

${ }^{2}$ Mucosal Immunization Research Group

Department of Microbiology

University of Alabama at Birmingham

Birmingham, Alabama 35294, USA 